\title{
'Equity smirks' and embedded options: the shape of a firm's value function
}

\author{
Adam Ostaszewski \\ Dept. of Mathematics \\ London School of Economics
}

July 14, 2004

\begin{abstract}
This paper examines the methodology and assumptions of Ashton, D., Cooke, T.,Tippett, M., Wang, P. (2004) employing recursion value $\eta$ as an explanatory single-variable in a model of the firm, first introduced by Ashton, D.,Cooke,T.,Tippett in (2003). A qualitative analysis of all of their numerical findings is given together with an indication of how more useful is the tool of special function theory, here requiring confluent hypergeometric functions associated with the Merton-style valuation equation

$$
\frac{1}{2} \zeta \eta \frac{d^{2} V}{d \eta^{2}}+(r-q) \eta \frac{d V}{d \eta}-r V=0
$$

A justification and a wider interpretation of their model and findings is offered: these come from inclusion of strictly convex dissipating frictions arising either as insurance costs, replacement costs of funds paid out, or of debt service, and from the inclusion of alternative adaptation options embedded in the equity value of a firm; these predict not only a J-shaped equity curve, but also, under the richer modelling assumption, a snakelike curve that may result from financial frictions like insurance. These 'smirks' in the equity curve may be empirically tested. It is shown that the inclusion of frictions in dividend selection (e.g. the signalling costs of Bhattacharya) leads to an optimal dividend payout of $\alpha \eta$ that is a constant coupon for an interval of $\eta$ values preceded by an interval in which $\alpha=r$; this is at variance with the ACTW model where the exogeneous assumption of a constant $\alpha$ is made.
\end{abstract}

This is the full, detailed, version of a discussion paper presented at State of the Art International Advances in Accounting Based Valuation at the Cass Business School, a conference held in association with the journal, Accounting \& Business Research, London, December 12th and 13th 2003. I am indebted to Ken Peasnell for suggesting the word 'smirk'. I am grateful to Jim Ohlson, Mark Tippett, Miles Gietzmann, to colleagues at LSE, and to colleagues at University College London Mathematics Department, particularly Susan Brown and S.N. Timoshin for very helpful discussions. 


\section{Introduction}

Ashton, D., Cooke, T.,Tippett, M. (2003) and Ashton, D.,Cooke, T.,Tippett, M.,Wang, P. (2004), henceforth referred to as ACTW, offer an elegant and ground-breaking continuous-time model for the evolution of accounting values over time, blending the approaches of Accounting Theory and Finance Theory. The significance of the analysis in ACTW is three-fold: firstly it gives an insight - through the prism of an accounting variable - into how a firm's dividend policy has a controlling role in the determination of equity value; secondly, it explains in terms of one type of embedded option an important empirical observation regarding the J-shaped functional relation between earnings and equity, a feature not explicitly modelled until quite recently - see also Gietzmann and Ostaszewski (2004); and thirdly (though this may be seen as secondary to the main thrust of the argument) it admits the so-called dirty surplus accounting systems (i.e. it allows earnings to be partitioned into exceptional and non-exceptional with both these evolving separately, though not necessarily independently).

The essential assumption of ACTW is the adoption of so-called recursionvalue, denoted by $\eta$, as the explanatory variable. This variable is at any time the expected present value of the future dividend stream, given the current information, and contingent on the assumption that the production technology/environment is to remain unchanged. They examine a specific stochastic model written in accounting terms, namely the natural, constant-matrix, continuous-time generalization of the Ohlson (1995) discrete-time linear model as governing the evolution of earnings, with uncertainty modelled by a squareroot process driven by $\eta$. Theirs is thus a state space model with

$$
d \mathbf{y}=C \mathbf{y} d t+\eta^{\delta} d \mathbf{z}_{t},
$$

where abnormal earnings $a$, dirty surplus adjustment $\varepsilon$, and one additional information variable $v$ make up the state vector at any instant, $\mathbf{y}_{t}$, while $\mathbf{z}_{t}$ is a vector of Brownian motions, and $\delta$ is assumed not to exceed $1 / 2$. The technology regime thus remains unchanged as long as the matrix $C$ at the heart of the model is constant. They also assume the firm has access to alternative modes of operation giving it what they term an 'adaptation value'. Further simplifying assumptions (several) lead them to an ordinary differential equation for the valuation of the firm and to a unique solution which exhibits the J-shape. Based on this solution function ACTW conduct comparative statics to show the influence of dividend policy over equity value.

The simplifying assumptions are: a single sde, a specific dividend policy and just one type of adaptation technology which is characteristic of a firm entering a contraction phase.

The first aim of the current paper is to widen the argument, so as to support the simplifying assumptions, given the remarkable insight ACTW offer. Such an argument is called for, since the assumptions are contentious on the grounds of an apparent inconsistency with the starting linear model. The second aim is to widen the perspective, so as to embrace a richer structure of embedded options, including not only contraction options but also expansion options; this opens up 
an analysis of firms according to their embedded option structure, and a realization that J-shapes are not the only shapes that may be observed; we indicate a contrasting 'snake-like' shape driven by a 'cost of finance' friction. A third aim is to offer two further simplifications: one in respect of the method employed for solving the underlying differential equation (in particular this insight rids the model of an unrealistic 'cusp-like' feature - a vertical tangency at the origin), but more significantly is more helpful to analyzing the solutions than the quadrature (which ACTW use to investigate their own integral representation of the solution); the other simplification is in respect of the comparative statics which are here derived from an even simpler perspective (a stripping down to an appropriate exponential decay, which is thus seen to be an important controlling feature of the value function). A final aim is to endogenize the choice of dividend policy. It will be clear from the analysis here that the next step in a research programme is to perform a taxonomy of switching models consistent with the multitude of qualitatively different solutions available by 'patching solutions' from a collection of broadly similar, underlying, differential equations.

The structure of this paper is the following. A brief description generalizing some aspects of the model is given first. Various consequences of the ACTW paradigm and an apparent inconsistency is analyzed as a thought experiment; then reference to the Bugstahler-Dichev (1997) model which inspires the ACTW paradigm indicates why, in principle, smooth-pasting of solutions from two differential equations is a must, and why, nonetheless, the simplification adopted by ACTW is justifiable. In section 4.2 a qualitative analysis of the single differential equation approach is given and it is shown that introduction of a friction term not only removes the 'cusp-like' feature on the short lip of the J-shape, but also permits a snake-like shape. Comparative statics from a simplified perspective are given in section 7 and these relate to issues of monotonicity (in $\mu$ ), location of the minimum equity value ('the bottom of the smirk'), and comparison against the non-dividend regime. The final section considers endogenizing the dividend policy when frictions are included; there is a growing literature concerned with dividend policy as a control variable and just a few recent papers are cited. The conclusion short-lists how this approach might throw further light on a more significant number of dividend-related questions. The Appendix collects proofs, and en passant, a brief summary of some pertinent facts concerning the solution of the confluent hypergeometric differential equation (which is at the heart of the ACTW model) including series as well as Laplace Transform approaches to the equation.

\section{A more general model}

ACTW have as a starting point the introduction of a continuous variable $\varepsilon(t)$ which is the instantaneous value of the so-called exceptional items (the "dirty surplus" adjustment) with the consequence that cost of capital $i$ is charged only to adjusted earnings (excess) earnings $x(t)$; residual income/abnormal earnings 
are thus defined instantaneously by

$$
a=x-i b,
$$

so that unadjusted earnings over an interval of length $\Delta t$ are $(x+\varepsilon) \Delta t$; here $i$ denotes the riskless interest rate, as in ACTW. The modelling of firm value proceeds by connecting the value of assets in hand (bookvalue, i.e. historic value of the assets held by the firm) and the expectation of the discounted stream of future dividends (see Miller-Modigliani (1961)) and begins by writing change in the bookvalue $b$ as a change in earnings less dividend payout:

$$
d b=(x+\varepsilon) d t-d D=(i b+a+\varepsilon) d t-d D,
$$

where $D=D(t)$ denotes at time $t$ the cumulative outflows from the firm (dividends in the ACTW case). Define

$$
\eta=E\left[\int_{0}^{\infty} e^{-i t} d D(t)\right]
$$

Absent any bubble phenomena at infinity, i.e. assuming

$$
\lim _{t \rightarrow \infty} e^{-i t} b_{t}=0
$$

integration by parts gives

$$
\begin{aligned}
\int_{0}^{\infty} e^{-i t} d D(t) & =\int_{0}^{\infty} e^{-i t}[i b+a+\varepsilon] d t-\int_{0}^{\infty} e^{-i t} d b \\
& =\int_{0}^{\infty} e^{-i t}[i b+a+\varepsilon] d t-\left(\left[e^{-i t} b\right]_{0}^{\infty}+i \int_{0}^{\infty} b e^{-i t} d t\right) \\
& =b_{0}+\int_{0}^{\infty} e^{-i t}[a+\varepsilon] d t
\end{aligned}
$$

The expected value on the left-hand side is interpreted as the recursion value of the firm denoted $\eta$ and this assumes the "firm's existing investment opportunity set will remain in force indefinitely" - with "opportunity set" defined by the modelling assumption of the linear system $C$ given by (1).

We begin by observing the following

Proposition 1. With $\eta$ defined by (3) and assuming the dynamics of bookvalue given by (2) satisfy (4), we have that

$$
E\left[d \eta_{t}\right]=i \eta_{t} d t-d D(t),
$$

provided the processes $(a(t), v(t), \epsilon(t))$ are continuous and $D(t)$ is non-anticipating, e.g. $d D(t)=f(\eta(t)) d t$ for some deterministic function $f($.$) .$

In particular, if $a$ and $\varepsilon$ are given by the linear stochastic differential equation (1), then for some combination $q$ of the original noise generators,

$$
d \eta=i \eta d t-d D+\zeta \eta^{\delta} d q
$$


Proof. See Appendix 1.

It follows that a stochastic model based on a standard Wiener process $z_{t}$ will take the form

$$
d \eta_{t}=i \eta_{t} d t-d D(t)+\zeta\left(\eta_{t}\right) d z_{t}
$$

and ACTW study the case

$$
\zeta(\eta)=\zeta_{0} \eta^{1 / 2}
$$

with $\zeta_{0}$ a positive constant. Their theorem identifies the constant $\zeta_{0}$ corresponding to the linear model (1) in terms of the Wiener processes generating $a(t), v(t), \epsilon(t)$.

We now consider a variant of the ACTW deterministic dividend policy in which the firm makes two payments: a constant coupon $C$ per unit time and a proportion $\alpha(\eta)$ of the recursive value $\eta$. The coupon may be thought of as a constant debt-service covenant, but other interpretations are also appropriate (see section 8); this component is absent in the ACTW model but we need it at two junctures, in particular to remove the 'cusp-like' phenomenon (infinite slope at the origin).

Assuming therefore that for all time

$$
d \eta=i \eta d t-d D+\zeta(\eta) d z
$$

and that for some deterministic function $\alpha($.$) it is the case that$

$$
d D(t)=[\alpha(\eta(t)) \eta+C] d t,
$$

then the equity value satisfies the equation

$$
\frac{1}{2} \zeta(\eta)^{2} \frac{d^{2} V}{d \eta^{2}}+(i \eta-\alpha \eta-C) \frac{d V}{d \eta}-i V+\alpha \eta+C=0 .
$$

Note that $V=\eta$ is a particular solution (as it must be in the absence of any switching options), so that the general solution is given by:

$$
V(\eta)=\eta+W(\eta)
$$

where $W$ solves the complementary equation

$$
\frac{1}{2} \zeta(\eta)^{2} \frac{d^{2} W}{d \eta^{2}}+((i-\alpha) \eta-C) \frac{d W}{d \eta}-i W=0 .
$$

This is a minor modification to a well-known argument attributed to Merton (1974); see also Black and Cox (1975).

For the case $\zeta(\eta)=\sqrt{\zeta \eta}$ with $\zeta$ a positive constant, rescaling the independent variable to $y=2 \lambda \eta$, where $\lambda=2(i-\alpha) / \zeta$ (assuming that $\alpha<i$ ), and tidying up of parameters (by setting $\kappa=i /(i-\alpha)$ and $c=2 C / \zeta$ ) we find that the format of the governing differential equation is thus

$$
y \frac{d^{2} W}{d y^{2}}+(y-c) \frac{d W}{d y}-\kappa W=0 .
$$


We will refer to this as the generalized Merton valuation equation. It may be solved by reference to the Kummer equation. The latter is obtained from (9) by setting $x=-y$ and $w(x)=W(y)$ and writing ${ }^{1} A=-\kappa$ and $B=-c$, so that

$$
x \frac{d^{2} w}{d x^{2}}+(B-x) \frac{d w}{d x}-A w=0 .
$$

There are a number of established special functions which may be used to solve the equation (10) and mention should be made in particular of the Kummer functions and of the Whittaker functions. See Slater (1960). In section 4.3 two independent solutions of (10) of financial significance are found to be given by the Kummer functions $M(A, B, x)$ and $U(A, B, x)$, so that the Merton valuation equation has the two solutions

$$
C(y)=M(-\kappa,-c,-y), \quad P(y)=U(-\kappa,-c,-y) .
$$

An alternative notation for $M(A, B, x)$ is ${ }_{1} F_{1}(A ; B ; x)$, a notation which refers to the coefficients in the hypergeometric series expansion of the Kummer function $M .^{2}$ The reader should be warned that in the domain of financial interest there are potential difficulties in accessing the special functions on computer (problems with integer values for $A, B)$. Some useful formulas are collected in Appendix 2 for ease of reference.

There is an immediate payoff to this extra level of generality: in the special case of $c=\kappa$ the equation (9) has an obvious put-like solution: $W(y)=e^{-y}$. Its call-like second solution is close to being a simple power function $y^{\kappa}$. See section 6. This makes the model a hybrid of the arithmetic Brownian (with exponential solution) and geometric-Brownian (with power solution).

\section{Consequences of aggregation and the simpli- fying paradigm}

ACTW derive two consequences of the modelling assumption (1).

The first is that in such a framework the current value of $\eta$ can be expressed in terms of book-value and the current state variables in the form

$$
\eta(t)=b(t)+H\left(\begin{array}{l}
a \\
v \\
\varepsilon
\end{array}\right)
$$

where $H=(1,0,1)(i I-C)^{-1}$ is a row vector computed from $C$. It is tacitly assumed that $i$ is not an eigenvalue of $C$. Though ACTW do not consider this

\footnotetext{
${ }^{1}$ Traditional notation, see Slater(1960), uses lower case letters which we avoid since $a, b$ already denote abnormal earnings and book-value.

${ }^{2}$ The $\mathrm{n}$-th term of the series ${ }_{p} F_{q}(\mathbf{a} ; \mathbf{b} ; t)$ is

$$
\frac{\left[a_{1}\left(a_{1}+1\right) \ldots\left(a_{1}+n-1\right)\right] \ldots\left[a_{p}\left(a_{p}+1\right) \ldots\left(a_{p}+n-1\right)\right]}{\left[b_{1}\left(b_{1}+1\right) \ldots\left(b_{1}+n-1\right)\right] \ldots\left[b_{q}\left(b_{q}+1\right) \ldots\left(b_{q}+n-1\right)\right]} \frac{t^{n}}{n !} .
$$
}


point, it is a justified enquiry, given that optimal activity is predicated on $\eta$ alone, whether, at least in principle, the state vector $(a(t), v(t), \epsilon(t))$ is recoverable from knowledge of $\eta$ obtained within some, or any (no matter even how short), time interval starting at $t$. Set the volatility to zero for a simpler and more achievable task and the answer is no. This makes the findings of ACTW all the more remarkable.

Proposition 2. The state vector $(a(t), v(t), \epsilon(t))$ of the linear system $C$ given by (1) is not recoverable from knowledge of $\eta$ obtained within some, or any (no matter even how short), time interval starting at $t$.

Proof. The reconstruction question has a well-known equivalent in the deterministic setting (see Russell (1979)); the equivalent statement is that the following matrix has rank 3 . We compute the form of the required matrix to be

$$
\begin{aligned}
{\left[\begin{array}{c}
H \\
H C \\
H C^{2}
\end{array}\right] } & =\left[\begin{array}{c}
(1,0,1)(i I-C)^{-1} \\
(1,0,1)(i I-C)^{-1} C \\
(1,0,1)(i I-C)^{-1} C^{2}
\end{array}\right]=\left[\begin{array}{c}
(1,0,1)(i I-C)^{-1} \\
(1,0,1)\left[i(i I-C)^{-1}-I\right] \\
(1,0,1)\left[i^{2}(i I-C)^{-1}-(1+i) I\right]
\end{array}\right] \\
= & {\left[\begin{array}{c}
H \\
i H-(1,0,1) \\
i^{2} H-(1+i)(1,0,1)
\end{array}\right], }
\end{aligned}
$$

since

$$
\begin{aligned}
(i I-C)^{-1} C & =i(i I-C)^{-1}-I \\
(i I-C)^{-1} C^{2} & =i(i I-C)^{-1} C-C=i^{2}(i I-C)^{-1}-i I-I
\end{aligned}
$$

However, this matrix cannot have rank 3 since after subtracting appropriate multiples of $H$ we obtain the equivalent matrix

$$
\left[\begin{array}{c}
H \\
(1,0,1) \\
(1+i)(1,0,1)
\end{array}\right],
$$

which has rank at most 2 .

Secondly, they show that since the stochastic driver for each accounting variable is a square-root process in $\eta$, it follows that the stochastic differential equation obeyed by $\eta$ is

$$
d \eta_{t}=i \eta_{t} d t-d D(t)+\zeta \eta^{1 / 2} d z_{t},
$$

where $i$ is the required rate of return on investment, $D(t)$ is the cumulative dividend, $\zeta$ is a positive volatility constant and $z_{t}$ is a Wiener process (derived as a linear combination of the Wiener processes driving the assumed linear system). The equation (12) is critical to the ground-breaking developments from here on.

ACTW at this point show that it is possible to abandon the state-space model of a firm, run essentially by noise, in favour of a managed firm. In the 
managed firm managers monitor at each moment the expected value of the sum total of discounted dividends, $\eta$, assuming no intervention, but may, if they so choose, intervene and so let the firm run under a different technology. This option to switch adds value to the firm, lifting it from the original $\eta$ to $V(\eta)$. ACTW assume that (12) holds for all time. Though they do not say this, the implication of the assumption is that even when the firm has switched to a different technology, at which stage the corresponding new variable $\eta$ might have evolved differently, they assume that volatility is unchanged. (The assumption thus excludes investment of liquidated value in government bonds of zero volatility, in favour of technologies more in keeping with the original model $C$.) They also assume that the firm pursues throughout time an exogeneously selected proportionate dividend policy whereby

$$
d D(t)=\alpha \cdot \eta d t,
$$

with $\alpha$ a constant. Assuming $0<\alpha<i$ this form of dividend ensures that the firm continues to have value (is not expected to go bankrupt). ACTW thus in effect posit that the infinitely-lived firm's value $V(\eta)$ is a Merton-style perpetual option written on $\eta$ with $\eta$ regarded as a traded asset and with price described by (12). This is an ordinary differential equation and is the time-invariant generalization of the Black-Scholes equation when inflows and outflows of cash occur in the firm. We will refer to it simply as the standard Merton valuation equation. Here it takes the form

$$
\frac{1}{2} \zeta \eta \frac{d^{2} V}{d \eta^{2}}+(i-\alpha) \eta \frac{d V}{d \eta}-i V+\alpha \eta=0 .
$$

ACTW go on to assume that the option to switch out of recurring activity is one which is only attractive at low values of $\eta$ (see below for details); this single assumption enables them to identify a unique function $P(\eta)$ such that $V(\eta)=$ $\eta+P(\eta)$ solves (14), so that $P(\eta)$ identifies the value of such an adaptation option. Let us call this real option, the adaptation put. Given that $\eta$ is a particular solution of (14), the function $P(\eta)$ is of course a solution to the complementary equation obtained from (14) by setting $V(\eta)=\eta+W(\eta)$, where

$$
\frac{1}{2} \zeta \eta \frac{d^{2} W}{d \eta^{2}}+(i-\alpha) \eta \frac{d W}{d \eta}-i W=0 .
$$

The assumptions which lead to a unique adaptation put may be likened to the cutting of the Gordian knot rather than to its unravelling. There is a devil of a potential over-simplification; yet in this special case the approach is instrumental to yielding a valuable insight. We offer explanations in the next sections, warning the reader that assumptions alternative to having (12) hold for all $\eta$ would lead to alternative adaptation put functions (especially so if the alternative is investment in high-grade bonds).

In summary: set aside any particular choice of linear model $C$ for the variables $a(t), v(t), \epsilon(t)$, and instead take as starting point (6); if we assume that 
(12) holds for some $\zeta=\zeta(\eta)$, then (14) describes the value of any claim on $\eta$. For the simplest model, with $\zeta$ a constant, it is possible to characterize all non-negative solutions. This is done in section 4.

\subsection{A thought experiment for and against the simplifica- tion paradigm}

We indulge in what is only a thought experiment to understand the status of the assumptions ACTW make. If the firm has one mode/technology of operation, say that described by $C$ above, then its equity value at any time $t$ is given by $\eta_{C}(t)$ (as a discounted sum of dividends) governed by (12) in which $\zeta=\zeta(C)$. Suppose that the firm has access to alternative technology to which it may switch its operation by transferring all resources to it, i.e. it may at a time of its own choosing replace $C$ by a matrix $\bar{C}$. This is supposed to be its 'adaptation technology'. In the new context its equity value, as determined by $\bar{C}$, is $\eta_{\bar{C}}(t)$ with a stochastic differential equation formally like (12) though in general with a different volatility $\zeta=\zeta(\bar{C})$. At this point it is worth referring to the paper by Radner and Shepp (1996), where switching to alternative technologies leads to a change both in drift and in volatility; but note that there the state variable is quite different, as it represents accumulated net revenue and allows for bankruptcy.

Returning to the thought experiment, if the switch occurs at a time $\theta$, when $\eta_{C}(\theta)$ first achieves some appropriate moving target $\bar{\eta}(t)$, the equity value thereafter is given by the process $\eta_{\bar{C}}(t)$ started at the target value (assuming no further switches are allowed). If the governing equation is still (12), i.e. $\zeta(C)=\zeta(\bar{C})$, then it is difficult to distinguish between the two modes of operation from only a knowledge of $\eta$, and the need for a switch is placed in doubt (particularly, as neither system is likely to be reconstructible from a knowledge of only $\eta$ ). Assume now that the volatilities are distinct. At any time $t$ the target value $\bar{\eta}(t)$ for switching must depend on all of $C, \bar{C}$, perhaps $\eta(t)$, as well possibly as $t$ (but given the infinite horizon and the stationary framework this should immediately be ignored), and presumably, though not necessarily, on the vector of state variables $(a(t), v(t), \varepsilon(t))$. This latter point is a subtle one, but ACTW have implicitly shown elsewhere (footnote 14 in Ashton, D.,Cooke,T.,Tippett (2003)) that the consequences of writing $V=V(b, a, v, \varepsilon ; C, \bar{C})$ is a valuation equation that is a pde in the variables $b, a, v, \varepsilon$ and that the assumption that this equation has a solution in the form $v=V(\eta, C, \bar{C})$, given the linear form (11), reduces the pde to the ode (14). An invocation of a uniqueness theorem (for the solution of the pde), then validates the intuition that $\eta(t)$ is enough! Hence the form of the optimal switching policy is that $\eta$ achieves a value $\bar{\eta}=\bar{\eta}(C, \bar{C})$.

So assuming it is enough to know $\eta$ to determine the optimal switching, the value of the firm at time $t$ takes the form $V(\eta(t))$. It follows that the functional equation

$$
V(\eta)=d D(t)+e^{-i d t} E[V(\eta+d \eta)),
$$

correctly describes the risk-neutral valuation $V$ of the dividend flow, $i$ being 
the required rate of return. To deduce a Merton valuation equation one in principle needs to assume as data a form for the volatility term on each side of the switching point $\bar{\eta}$. Writing this in the form $\zeta=\zeta(\eta)$ and expanding (16) via Itô's Formula then gives the Dynkin-Kolmogorov differential equation (see Oksendal (1998) satisfied by $V$ to be

$$
\frac{1}{2} \zeta(\eta)^{2} \frac{d^{2} V}{d \eta^{2}}+(i-\alpha) \eta \frac{d V}{d \eta}-i V+\alpha \eta=0,
$$

compare Radner and Shepp (1996).

ACTW side-step this kind of thought experiment and simply posit that the condition (16) holds alongside of (12) and (13); the upshot of these is that $\zeta(\eta)$ is taken to be a constant multiple of $\eta$ (i.e. either as though changes in $\zeta$ for larger $\eta$ are largegly irrelevant as regards the qualitative behaviour, or as though $\zeta(C)=\zeta(\bar{C})$ which might at best be regarded as a limiting scenario). ACTW thus arrive at the Merton valuation equation in the form (14). We will see from later sections that little is lost as a result of the simplifying assumption insofar as the qualitative features of the associated put option are concerned.

\subsection{The Burgstahler-Dichev paradigm: smooth pasting}

Though the ACTW approach is motivated by the Burgstahler-Dichev approach (1997) to firm value, it should be regarded as a generalization not of the Burgstahler-Dichev call option paradigm, but of the Merton perpetual putoption paradigm as we show below. This involves smooth pasting a timeindependent solution of the Black-Scholes equation onto the exercise value of a put. By passing to a square-root process ACTW alter the geometry significantly enough to agree with empirical findings. However they side-step the need for a smooth-pasting between the exercise value and waiting value by assuming that both regimes are described by one equation. We will show that this rolling together leads to no loss in qualitative behaviour.

Burgstahler-Dichev posited a convex, increasing functional relation between earnings and market value, modelled after Black-Scholes as a call option on the next period earnings. Grounds for this latter view on value is that the firm exercises a call-like real-option in the form of a simple switching opportunity. The interpretation is that either the firm may stick with their recurring activity (which is assumed to offer a future value proportionate to earnings), or abandon this in the very next period. It would opt for abandonment, if the future value of recurring activity is too low, as compared to 'adapting' their behaviour to a superior value. This latter action, however, offers only a flat (constant) value over the whole range of earnings, and so is only superior at low earnings. We note that this corresponds to taking $\eta=K=$ const as the governing equation for low $\eta$ in lieu of (12).

Empirical tests found justification in the data for convexity, but surprisingly detected an initial portion of the functional relation to be downward sloping rather than flat (a portion corresponding to an up-turn in value as earnings fall). 
Of course our summary of the Burgstahler-Dichev approach shows it to be in reality a two-period model since it is inconceivable that the recurrent activity should give a linear future value curve. ACTW side-step a proper inductive (=recursive) treatment of the Burghstaler-Dichev paradigm in discrete time, by passing to continuous time where the Bellman equation, in this case an ordinary differential equation, replaces the induction step.

A review of the well-known perpetual put argument is profitable here. This argument is in continuous time, so different in flavour from that of BurgstahlerDichev as summarized above. Suppose given a fixed dividend payout rate $\alpha \eta$, then the following stochastic differential equation is consistent with Proposition 1 above, namely

$$
d \eta_{t}=(i-\alpha) \eta d t+\sigma \eta d z_{t} .
$$

A volatility term of $\sigma \eta$ is ruled out for ACTW if the equation is to hold for all time (all $\eta$ ), since they seek a put-like solution, and in the case $\zeta(\eta)=\sigma \eta$ the put-like solution has, as is well-known, a sigularity (blow up) at the origin. For the present pasting purposes, however, it is a perfectly admissible alternative to $\zeta^{1 / 2} \eta^{1 / 2}$, as long as this volatility form is applicable away from zero by the exercise of an alternative choice of technology near the origin. The equation (17) treats $\eta$ just like a standard dividend-paying traded asset. At any future moment in time, management have an American perpetual option: either to receive a value $K$ (by switching to the adaptation technology), or to continue with the recurring activity (and so holding onto an asset worth $\eta$ ). If $\eta>K$ there is no value to be had from exercise, and so the current value of the firm is $\eta$; but for $\eta<K$, one should consider exercise of the switching option in order to receive a value $V=K=\eta+(K-\eta)^{+}$. The optimal trigger for switching, $\bar{\eta}$, is well-known to be characterized here by the Black-Scholes equation for the perpetual put $P=(K-\eta)^{+}$, namely

$$
\frac{1}{2} \sigma^{2} \eta^{2} \frac{d^{2} P}{d \eta^{2}}+(i-\alpha) \eta \frac{d P}{d \eta}-i P=0,
$$

insofar as it stays valid for $\eta>\bar{\eta}$ (whilst the put remains unexercised), alongside the smooth-pasting condition: $P^{\prime}(\bar{\eta})=-1$. The latter condition may be viewed as a pasting together of two solutions: one from the Black-Scholes equation (18) and the other from the more trivial differential equation $P^{\prime}(\eta)=-1$ (obeyed by $P=K-\eta)$; in addition the pasting requires continuity of both the value function $P$ and its derivative ${ }^{3} P^{\prime}$. The solution function for $\eta>\bar{\eta}$ is a declining

\footnotetext{
${ }^{3}$ The usual considerations apply to the two differential equations. Suppose $P_{0}(\eta)$ is the appropriate value function for $\eta$ in $(0, c)$ obtained from one d.e. Then the solution $P(\eta, c)$ obtained by solving the other d.e. to the right of $\eta=c$ is subject to inter alia the boundary condition $P(c, c)=P_{0}(c)$. That yields on differentiation

$$
P_{0}^{\prime}(c)=P_{\eta}(c, c)+P_{c}(c, c)=P_{\eta}(c, c) .
$$

Indeed the optimal choice of $c$ is to maximise option-value and this requires at $\eta=c$ the maximisation of $P(\eta, c)$, i.e. that $P(c, c+h)$ is to be maximised at $h=0$.
} 
inverse power function (with the power typically below unity ${ }^{4}$ ) albeit with an initial linear portion of slope -1 . It is the linear portion which produces a flat initial portion for $V(\eta)=\eta+P(\eta)$, at odds with empirical findings which exhibit an up-turn on approach to the origin.

ACTW move away from the geometric Brownian model to a square rootprocess and ditch the patching of two solutions from two differential equations; they thus obtain a put $P(\eta)$ that is 'in the large' a product of a not too dissimilar inverse power ${ }^{5}$ and a decaying exponential solution. Close to the origin the option value is finite (although its slope is large, in the limit it is $-\infty$ ) and so the put is now consistent with the empirical finding. It is possible (see section 5) to bring down the infinite slope to a more meaningful finite value by introducing financial frictions.

A more general application of the ACTW approach would patch two qualitatively similar put-like functions obtained from two different but similarly structured differential equations; but, despite the additional intellectual cost, this would achieve qualitatively the same result as from the simplifying ACTW assumption.

\section{Valuation equation: a qualitative analysis}

ACTW obtain an integral representation of the solution of the Merton valuation equation (14) and use numerical integration in order (i) to plot the adaptation value against $\eta$ and (ii) to perform comparative statics in relation to varying the dividend policy. For this approach asymptotic considerations are a technical necessity. However an alternative route is proposed here based on qualitative considerations of the ode and on series expansions of the solution; these seem more appropriate, since much interest centers on values of $\eta$ near the origin. Some simple manipulations routine to the theory of differential equations offer key insights (and a route to a valid proof). Consideration of the comparative statics is delayed to section 7 .

One's first focus of attention is to check whether there are two independent solutions (as (15) is of second order) capable of being fundamental from a financial viewpoint. One expects following the classic example of Merton-style perpetual options, see Merton (1970), to have a put-like solution as well as a call-like solution.

\footnotetext{
${ }^{4}$ Letting

$$
\rho=\frac{2 i}{\sigma^{2}}, \delta=\frac{2 \alpha}{\sigma^{2}}
$$

and for simplicity setting $\rho=1$, an acceptable bench-mark value, we get the negative power to be $-\beta$ where

$$
\beta=\sqrt{1+(\delta / 2)^{2}}-\frac{\delta}{2}=1-\frac{1}{2} \delta+\frac{\delta^{2}}{8}+\ldots
$$

which for small $\delta$ is below unity.

${ }^{5}$ The power will be numerically somewhat greater if we again take the dimensionless 'required rate' at its benchmark value of unity and assume a small dimensionless dividend rate. Here the power will be $-\kappa=i /(i-\alpha)$, or around $-(1+\delta)$, as opposed to $-1+\delta / 2$.
} 


\subsection{Tidying up to get Kummer's equation}

Introduce the notation

$$
\lambda=\frac{i-\alpha}{\zeta}>0, \quad \mu=\frac{i}{\zeta}>0, \quad \kappa=\frac{\mu}{\lambda}=\frac{i}{i-\alpha} \geq 1,
$$

so that in particular the dividend rate relative to the required rate, namely $\alpha / i$, is $(\kappa-1) / \kappa$. Now $(15)$ becomes

$$
W^{\prime \prime}(\eta)+2 \lambda W^{\prime}(\eta)-\frac{2 \mu}{\eta} W(\eta)=0,
$$

and if $\lambda>0$ the scale change $y=2 \lambda \eta$ is appropriate so that putting $W(\eta)=$ $Q(y)=Q(2 \lambda \eta)$, yields

$$
y Q^{\prime \prime}+y Q^{\prime}-\kappa Q=0 .
$$

The integer values $\kappa=1,2$ are considered in ACTW. The case $\lambda=0$ is considered in Appendix 3. A further variable change, unexpected for the present financial context ${ }^{6}$, namely $x=-y$ and $Q(y)=U(x)=U(-y)$, leads to

$$
x U^{\prime \prime}-x U^{\prime}+\kappa U=0,
$$

a case of the well-studied confluent hypergeometric equation (or, Kummer's equation):

$$
x y^{\prime \prime}+(B-x) y^{\prime}-A y=0,
$$

quoting temporarily the notation after Slater (1960) to which we refer for a full treatment of this equation. The theoretical considerations shown there work at their smoothest when $A$ and $B$ avoid certain integer values, so that integer values for $A$ and $B$ require special fixes. From the finance perspective these are however values of interest (indeed in ACTW we have $B=0$ as well as $A=-1,-2)$. Salient features are collected below in Appendix 2 .

The conclusion is that special function theory (and the confluent hypergeometric functions are part of the menagerie) is here to stay and help. So one naturally asks for the significance of a non-zero $B$ parameter. This corresponds to a constant inflow or outflow of value on top of the dividend, possibly from frictions like costs or debt service (see section 8 ).

\subsection{Qualitative behaviour: puts, calls and the smirk}

The differential equation (20) may also be usefully transformed by setting

$$
W=e^{-\lambda \eta} Q
$$

to the normalized form (i.e. from which the first derivative is absent, see e.g. Ince (1956: 394), or Slater (1960))

$$
Q^{\prime \prime}=\left(\lambda^{2}+\frac{2 \mu}{\eta}\right) Q
$$

\footnotetext{
${ }^{6}$ It is motivated by complex variable considerations.
} 
a form much like that studied by Whittaker. (See Whittaker and Watson (1946).) Hence positive solutions $Q$ (if any) are convex for $\eta>0$ and there are two asymptotic exponential forms $e^{ \pm \lambda \eta}$ for large $\eta$. The insight that positive solutions of (20) are also convex is immediate but the argument is not fully accurate and requires an overlay based on the observation that $Q$ may only have local minima for $Q>0$ and only local maxima if $Q<0$. (This argument counters potential undesirable effects that the transforming exponential factor from $Q$ to $W$ might have.) This overlay also shows that positive solutions do exist with $Q(0)>0$, since unbounded positive solutions exist for large $\eta$, and such solutions cannot have a negative local minimum. For fixed $\kappa, \lambda$ the solutions form a one-parameter family (up to a scalar multiplier) and so the family of positive solutions with $Q(0)>0$ will contain one member with its minimum at infinity.

In summary we have the following characterization theorem.

Proposition Any solution of the linear differential equation (20) with $\lambda, \mu>$ 0 satisfying $W(0)>0$ and $W(+\infty)=0$ is a strictly convex decreasing function asymptotically of the form

$$
A \eta^{-\kappa} e^{-2 \lambda \eta}\left\{1+O\left(\eta^{-1}\right)\right\}
$$

for some positive constant $A$.

Positive solutions of the equation, i.e. with $W(0)>0$, have $W^{\prime}(0+)=-\infty$ (a 'cusp-like' feature) and have in general one local minimum possibly at infinity after which they are monotonic, increasing, unbounded and of the form

$$
A \eta^{\kappa}\left\{1+O\left(\eta^{-1}\right)\right\}
$$

the exception is the one decreasing function asymptotic to zero as $\eta \rightarrow \infty$ (whose local mimum is at infinity).

Non-negative solutions of the equation with $W(0)=0$ are monotonic, increasing, unbounded and of the form

$$
A \eta^{\kappa}\left\{1+O\left(\eta^{-1}\right)\right\}
$$

Proof. The asymptotic results follow from corresponding assertions regarding the Whittaker functions. See Whittaker and Watson (1946).

Note that in this last result for large $\eta$ it is plausible that the volatility should play an insignificant role in determining equity value. The effect on (15) of setting $\zeta=0$ is the corresponding first-order equation

$$
\eta \frac{d W}{d \eta}=\frac{i}{i-\alpha} W=\kappa W,
$$

whose solutions are multiples of

$$
W=\eta^{\kappa},
$$

confirming the Proposition. 
The proposition confirms that there are two independent monotonic solutions, one which is put-like, being decreasing and zero at infinity, which we might denote $W(\eta)=P_{\kappa}(2 \lambda \eta)$, identified by requiring additionally that $W(0)=1$, and the other, which is call-like, since it is zero at the origin, increasing and unbounded, which we might denote $W(\eta)=C_{\kappa}(2 \lambda \eta)$ identified by requiring additionally that $W^{\prime}(0)=1$.

A stronger conclusion follows in regard to the J-shaped equity value: the equity 'smirk' subsists in all of these non-negative solutions, not merely in the put-like case.

Remark. The proposition extends to the solution functions of the general valuation equation (9) provided the coupon is not too large; for this extension it is sufficent that

$$
c<2 \kappa \text {. }
$$

Indeed the normalized form for

$$
y \frac{d^{2} W}{d y^{2}}+(y-c) \frac{d W}{d y}-\kappa W=0 .
$$

is

$$
Q^{\prime \prime}(x)=J(x) Q(x)
$$

where

$$
J(x)=\frac{1}{4}+\left(\kappa-\frac{1}{2} c\right) \frac{1}{x}+\frac{1}{4}\left((c+1)^{2}-1\right) \frac{1}{x^{2}} .
$$

For details see Bender and Orszag (1999) page 88 (Schrődinger equation), or Miller (1950).

\subsection{The fundamental solutions}

Both these positive solutions are in the classical menagerie of special functions and may be computed using infinite series and the logarithm function (see Appendix 2). Alternatively they may be accessed by Laplace Transforms (see Appendix 5). The put-like option is decreasing and is attractive when small earnings are indicated by $\eta$, so corresponds to a firm's option for entering contraction in their activity; the other is increasing and is increasingly attractive when large earnings are indicated by $\eta$ so corresponds to expansion. A second order equation in one variable permits therefore two kinds of adaptation. ACTW focus on the decreasing put-like option $P_{\kappa}(\eta)$ which is quite right if one wishes to recreate an analogue to Bugstahler and Dichev's constant value option. In consequence ACTW ignore the more general circumstances where a company may also have multiple options. In general the model predicts a valuation of the form

$$
V(\eta)=\eta+A \cdot P_{\kappa}(2 \lambda \eta)+B \cdot C_{\kappa}(2 \lambda \eta)
$$

This should be compared and contrasted with the microeconomic model of Gietzmann and Ostaszewski (2004) where there are three options, in general: to 
contract, to expand and to partition inputs across time, and unsurprisingly there are two state variables (if time is disregarded).

As for the functions $C_{\kappa}(x)$ these take a gratifyingly simple form being polynomials of degree $\kappa$ for integer values of $\kappa$, where the values $1,2,3$ etc correspond respectively to $\alpha=0, i / 2, \ldots$ i.e. zero, half the required rate of return dividends, two-thirds etc (since $\alpha / i=(\kappa-1) / \kappa)$. They are proportional to (are multiples of) the generalized Laguerre polynomials, (Abramowitz and Stegun (1972: 778), $\kappa ! L_{\kappa}^{(-1)}(-x)$ of degree $\left.\kappa\right)$, namely:

$$
C_{1}(x)=x, \quad C_{2}(x)=x^{2}+2 x, \ldots
$$

These functions have value zero at the origin, as are all the increasing solutions for $\kappa$ non-integral. They are accessible through Mathematica as a multiple by $(-1)^{\kappa}(\kappa !)$ of HypergeometricU $[-\kappa, 0,-z]$, or as a multiple by $(\kappa-1)$ ! of Hypergeometric1F1Regularized $[-\kappa, 0,-z]$.

The corresponding second solutions for $\kappa=1$ and $\kappa=2$ are available in the logarithmic format

$$
\begin{aligned}
U_{1}(x, \beta) & =1+\beta x-\frac{1}{2} x^{2}\left\{1-\frac{1}{6} x+\frac{1}{6} x^{2}-\ldots\right\}+x \ln x, \\
U_{2}(x, \beta) & =1+\beta x+\frac{1}{2}(\beta-3) x^{2}-\frac{1}{6} x^{3}\left\{1-\frac{1}{6} x+\frac{1}{6} x^{2}-\ldots\right\}+\left(x^{2}+2 x\right) \ln x \\
& =1+\frac{\beta}{2}\left(x^{2}+2 x\right)-\frac{3}{2} x^{2}-\frac{1}{6} x^{3}\left\{1-\frac{1}{6} x+\frac{1}{60} x^{2}-\ldots\right\}+\left(x^{2}+2 x\right) \ln x,
\end{aligned}
$$

where for $\kappa=1$ the choice $\beta=-.4227846$ yields $P_{1}(x)$ and in the case $\kappa=2$ the choice $\beta=1.1544314$ yields $P_{2}(x)$.

In general for $\kappa$ an integer we have for some constant $\beta$ and a standard polynomial $B_{\kappa}(x)$ of degree $\kappa$ (details in Appendix 2) that

$$
v_{\kappa}(x)=C_{\kappa}(x) \ln x+\beta \cdot B_{\kappa}(x)-\frac{a_{1}}{\kappa(\kappa+1)} x^{\kappa+1}\left\{1-\frac{1}{(\kappa+1)(\kappa+2)} x^{2}+\ldots\right\} .
$$

The case $\kappa=\infty$ corresponding to $\alpha=i$ (where $\lambda=0$ ) is relevant when the dividend rate is to be bounded above on the grounds of solvency. The solution function is given by modified Bessel functions of order one and is considered in Appendix 5.3.

Remark on stability. A small perturbation in the value of $\beta$ will alter the behaviour of the second solution, so that it either becomes unbounded, and either positive and increasing, or ultimately negative. Restricting the embedded options to the contraction activity only lays the ACTW model open to parameter estimation instability. Allowing alternative options would rescue the model from criticism.

\section{Cusp-like feature: removal by the coupon}

For a general positive coupon (non-integer) the first and second solutions have finite slope at the origin. Indeed in the case of the put-like solution this follows 
from the differential equation (8) directly since

$$
P^{\prime}(0)=-i P(0) / C=-i / C \text {. }
$$

Evidently as $C \rightarrow 0$ the slope becomes infinite.

Integral coupons may be of interest, so consider by way of example what happens when $b=-1$, i.e. $c=1$ (meaning $C=\zeta / 2$ ). Here for $\lambda>0$ in the original domain, the solution is $y(2 \lambda \eta)$ which for $\kappa>1$ comes in the form

$$
\begin{aligned}
y(x)= & \frac{\kappa(\kappa-1)}{2}\left(x^{2}+\frac{\kappa-2}{3} x^{3}+\ldots\right) \log x \\
& +1-\kappa x+b_{2}\left(x^{2}+\frac{\kappa-2}{3} x^{3}+\ldots\right)+\frac{\kappa(\kappa-1)}{2}\left(\frac{11+2 \kappa}{9} x^{3}+\ldots\right)
\end{aligned}
$$

and there is still no cusp-like feature at the origin. Moreover the function is not zero at the origin.

\section{A snake-shaped fund and the dissipation con- jecture}

In seeking to explain how both book value and capitalized earnings should enter into the valuation of the firm Burgstahler (1998) formulated two conjectures to be settled by empirical investigation. One is the convex shaped graph for equity value arising from the adaptation conjecture (which exercised us in the earlier sections above). The alternative is the dissipation conjecture, which assumes that differences between capitalized earnings and book value tend to be dissipated by economic forces; this is turned into a geometric statement to the effect that the value function may be represented by an increasing snakelike graph ${ }^{7}$; Burgstahler argues that under the dissipation argument "the market value falls below book value when capitalized earnings is below book value, and the market value falls above book value when capitalized earnings is above book value."

In this section we show that the generalized model of section 2 can also produce the snake-like shape for the value of a firm, but for rather different reasons which remain consistent with, rather than opposed to, the option-like foundation of the adaptation conjecture. Indeed a non-smooth linearized version of the dissipation shape may be synthesised by holding long a bond plus a call, and shorting a put with a strike set quite artificially below that of the call. Our contribution is to observe that a smooth analogue of this phenomenon may be naturally (rather than artificially) recreated using the generalized model of section 2 by using the naturally occurring put-like and call-like valuations associated with the embedded options of a firm.

\footnotetext{
${ }^{7} \mathrm{I}$ am grateful to Jim Ohlson for alerting me to the possible occurrence of such shapes and to Mark Tippett for this reference.
} 
We note in passing that one intuition for creating a 'holding company', or fund with such a portfolio, is an attempt to make gains from valuation mismatches arising from the application of what Abel, Dixit, Eberley and Pindyck (1996) term the 'naive investment rule' (whereby the current unit cost of capital is regarded as identifying the marginal value of capital installed in an enterprise). They identify the true relation between the marginal value of capital already in place in an enterprise computed without future investment/divestment adjustments (say $M V^{-}$) to the current unit cost of capital $p$ less the discounted maginal option values to expand or contract, by referring to these embedded options. In symbols the relation is that

$$
M V^{-}=p-\frac{P}{1+i}+\frac{C}{1+i}
$$

Thus a rationally held belief (e.g. by reason of some inertia) that $M V^{-}=p$ ignores (strategic) option values available to the enterprise through its ability to contract or expand in a future period.

As for the technicalities of our construction, begin by noticing that if $c=\kappa$ in the complementary equation (9), then one solution is $W(y)=e^{-y}$ and is put-like. If $c=\kappa=m$ another solution is identified ${ }^{8}$ in Appendix 2 as the polynomial

$$
u_{m}(y)=1-y+\frac{y^{2}}{2 !}+\ldots+\frac{(-y)^{m}}{m !},
$$

the partial sum to order $m$ of the series for $e^{-y}$. Note that for $m$ odd this function changes sign, so is not put-like or call-like. However, $C_{m}(y)=W(y)-u_{m}(y)$ is positive for $m$ odd and negative for $m$ even $^{9}$. It is thus call-like for $m$ odd $^{10}$ and by Taylor's Theorem behaves rather like the power function $y^{m+1}$. (The behaviour for $m$ even of the negative $-C_{m}(y)$ is analogous, and is in particular call-like.)

Combining with the particular solution $\eta=y / 2 \lambda$ we thus have the general form of the solution to the equity value equation (7) in the scaled variable $y$ as

$$
V(y)=\frac{y}{2 \lambda}+K e^{-y}+L\left(1-y+\frac{y^{2}}{2 !}+\ldots+\frac{(-y)^{m}}{m !}\right)
$$

for arbitrary $K$ and $L$. In the case $\kappa=3$ we can arrange for the function $V(y)$ to exhibit a monotonically decreasing snake-like shape involving a stationary inflection got by putting $K=L=1$ with an appropriate positive choice of $\lambda$. See Figure 1. Of course in view of the identity $W+u=2 W-(W-u)$, the value

\footnotetext{
${ }^{8}$ This solution may be found by the method of reduction of order. The substitution $u(y)=$ $v(y) e^{y}$ leads to a first order differential equation in $v^{\prime}$ which yields $u$ as a multiple of the integral$$
I_{m}=e^{-y} \int y^{m} e^{y} d y
$$

Indeed $u_{m}(y)=(-1)^{m} I_{m} / m !$.

${ }^{9}$ The partial sums of the alternating series for $e^{-y}$ overestimate for $m$ odd and underestimate for $m$ even.

${ }^{10}$ The function $C_{m}(y)$ has derivative $-C_{m-1}(y)$ so for $m$ odd is increasing.
} 


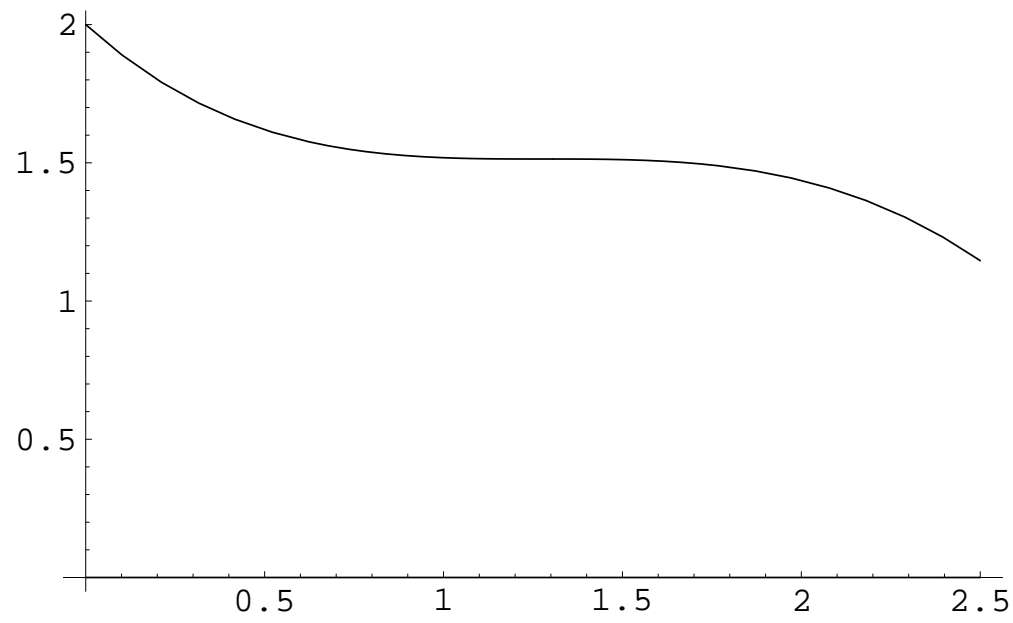

may be construed as a financial instrument (with underlying asset - equity in the firm) whose structure involves the firm as it is (valued at $\eta$ ), plus a long put (on its adaptation value), plus a short call on the firm. This shape may then be transformed to a positive, monotonically increasing, snake-like shape by a shift and a sign reversal, as the value of a portfolio/fund long on a bond, short on the firm with adaptation value and long on a call on the firm (to take advantage of expansion potential in the firm).

Note that to find a stationary inflexion point from $V(y)$ we need the firstorder condition

$$
\frac{1}{2 \lambda}-K e^{-y}-L\left(1-y+\ldots-\frac{(-y)^{m-1}}{(m-1) !}\right)=0,
$$

and the inflexion condition

$$
K e^{-y}+L\left(1+\ldots+\frac{(-y)^{m-2}}{(m-2) !}\right)=0
$$

to hold simultaneously. In the case when $m=3$ we have

$$
\frac{K}{L} e^{-1}=(y-1) e^{(y-1)}=W^{-1}(y-1),
$$

using the product-log function $W($.$) . Thus the location of the stationary inflex-$ ion point is

$$
y=1+W\left(\frac{K}{L} e^{-1}\right) .
$$

Choosing $K=L=1$ yields a value $y=1.278465$ from which we may choose a value of $\lambda$ via (24) to obtain the graph shown. 


\section{Comparative statics: a simplified perspective}

In this section a simplified function substitutes for the equity value; it is shown that the qualitative features considered by $\mathrm{ACTW}$ can be deduced from this simpler function.

\subsection{Monotonicity in $\mu$}

Recalling that $\lambda=\mu / \kappa$ and fixing $\kappa$ write the adaptation option in the form

$$
P_{\kappa}(\eta ; \mu)=\exp (-2 \mu \eta / \kappa) \text {. }
$$

Thus for fixed $\eta$ the adaptation value decreases as $\mu$ increases

\subsection{Bottoming out the Smirk (Location of the minimum)}

Using an asymptotic formula as a simplification now gives

$$
P(\eta)=\eta+P_{1}(0) e^{-2 \lambda \eta}
$$

which has a minimum (bottom of the 'smirk') when

$$
1=2 \lambda P_{1}(0) e^{-2 \lambda \eta}
$$

so

$$
\eta=\frac{\log 2 \lambda+\log P_{1}(0)}{2 \lambda}
$$

or

$$
\eta=(i-\alpha) \frac{\log 2+\log (i-\alpha)-\log \zeta+\log P_{1}(0)}{2 \zeta} .
$$

In the examples of $\mathrm{ACTW} 2 \lambda=\mu$ and $P_{1}(0)=1$, so the smile location is not available as a positive number; however with a larger dormant value, say with $P_{1}(0)>1 / \mu$ the smirk location reappears as a positive number.

\subsection{Comparison to a non-dividend paying firm.}

Using the simplification as earlier, in the case of the dividend-paying firm the put-like value is taken to be

$$
P_{\kappa}(\eta)=P(0) \exp (-2 \lambda \eta)
$$

whereas for the non-dividend firm one has

$$
P_{\kappa}(\eta)=P(0) \exp (-2 \mu \eta)
$$

To graph the specific example when $\mu=2 \lambda$ (i.e. $\kappa=2$ ), it is enough to look under a rescaling at $e^{-x}-e^{-2 x}$. The latter is non-negative function which is zero at the origin and at infinity, so will possess a maximum. See Figure 2. 


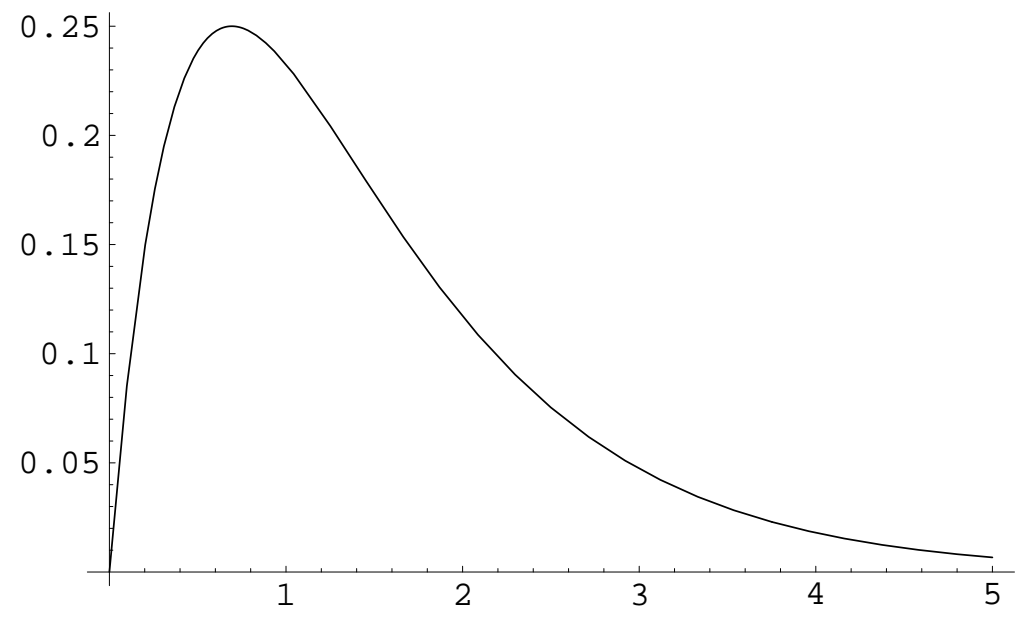

Evidently, since equity value is given by adding in $\eta$ to both firm types, the difference in their equity values is thus proportional to

$$
D(\eta)=e^{-2 \lambda \eta}-e^{-2 \mu \eta}>0 .
$$

This has a maximum when

$$
\mu e^{-2 \mu \eta}=\lambda e^{-2 \lambda \eta}
$$

or

$$
\frac{1}{1-\alpha / i}=\kappa=\frac{\mu}{\lambda}=e^{2(\mu-\lambda) \eta}=e^{2 \alpha \eta / \zeta}
$$

i.e.

$$
\eta=\frac{\zeta}{2 \alpha} \ln \kappa=-\frac{\zeta}{2 \alpha} \ln \left(1-\frac{\alpha}{i}\right)
$$

For one example considered by ACTW, in which $\zeta / \alpha=8$ and $\alpha / i=1 / 2$, this formula gives

$$
\eta=4 \log 2=2.77
$$

which over-estimates the peak position of 1.23. In the other example considered by ACTW, namely $\zeta / \alpha=4$ and $\alpha / i=1 / 2$, the formula gives

$$
\eta=2 \log 2=1.38
$$

which over-estimates the peak position of 1.28. Thus, for smaller values of $\zeta / \alpha$, the approximation improves.

Remark. In the presence of frictions, such as costs borne as a result of dividend payouts, it might no longer be true that the dividend-paying firm does better than its non-dividend paying counterpart. We note that the effect on equity value of the dividend payment is low when $\eta$ is low and when $\eta$ is large. 


\section{Varying dividend regimes}

This section is concerned with a variation of the ACTW model in which the choice of $\alpha$ in the dividend rate $\alpha \cdot \eta$ is endogenized; the analysis here has limited scope and in principle one should study other classes of dividend policies not just the proportionate ones (compare Jeanblanc-Picque-Shiryaev (1995) where bounded dividend policies are discussed, and creaming-off policies in which accumulation of reserves up to a threshold can be optimal; these approaches would be appropriate in the presence of call-like options but these are ignored here). For simplicity, it is assumed below that there is a cost incurred from paying the dividend rate $\alpha(\eta) \eta$ and that the cost, which is charged against the firm simultaneously with the payout, is at a rate $C(\alpha(\eta) \eta) i$. Two justifications for this friction come to mind. The first is a precautionary need by the firm to buy insurance against a fall in the value of $\eta$. However, in this case firms may wish to not insure at larger values of $\eta$ (in this case the costs are linear - caused by taxation losses), compare Rochet and Villeneuve (2004), and this issue is not studied here. An alternative reason is informed by the Bhattacharya (1979) model of a rationally reinvesting firm that is concerned with "making up shortfalls of cash flows relative to promised dividends", for which "the signalling benefit of dividends derives from the rise in liquidation value $V(D)$ caused by a committed, and actually paid, dividend level $D$ ". In the subsequent one-period Bhattacharya deduces a quadratic signalling cost when the end-of-period earnings $X$ have a general cumulative distribution $F(X)$ over a finite interval, and there is an additional constant cost rate $\gamma \geq 0$ of replacing required capital when the earnings fall below the dividend already paid out. His formula $C(D)$ for the cost of issuing $D$ is

$$
C(D)=\tau D+\gamma \int_{0}^{D} F(x) d x
$$

where $\tau$ is the income tax rate. Thus

$$
C^{\prime \prime}(D)=\gamma f(D) \geq 0
$$

and in general $C(D)$ is strictly convex; for earnings that are distributed uniformly on the unit interval this cost is ${ }^{11}$ :

$$
\tau D+\frac{\gamma}{2} D^{2}
$$

Though the distribution for $\eta$ is not uniform, for illustrative purposes the example below uses the simplest convex cost function, namely Bhattacharya's quadratic.

\footnotetext{
${ }^{11}$ At it simplest, earnings $x$ which at the end of one period are drawn uniformly from the unit interval have expected value $1 / 2$. If $D$ has been paid at the beginning of the period then assuming zero capital gains tax and an income tax rate of $\tau$, the expected loss to the equity holders arising from taxes on $D$ and the cost of replacing funds dropping below $D$ is

$$
\tau D+\int_{0}^{D} \gamma(D-x) d x=\tau D+\frac{\gamma}{2} D^{2} .
$$
}


Treating $\eta$ like a state variable governed by the state equation (15) and interpreting $d D$ now as the net outflow, so that

$$
d D(t)=\alpha \cdot \eta_{t} d t-C\left(\alpha \cdot \eta_{t}\right) i d t
$$

it follows that

$$
\begin{aligned}
d \eta_{t} & =i \eta_{t} d t+\zeta^{1 / 2} \eta^{1 / 2} d z_{t}-d D \\
& =\left((i-\alpha) \eta_{t}+C\left(\alpha \cdot \eta_{t}\right) i\right) d t+\zeta^{1 / 2} \eta^{1 / 2} d z_{t}
\end{aligned}
$$

So supposing that $\alpha=\alpha(\eta)$ is a control variable selected to maximize

$$
V(\eta)=E\left[\int_{0}^{\infty} e^{-i t} d D(t) \mid \eta_{0}=\eta\right]
$$

the Bellman equation may be written in the form:

$$
\begin{aligned}
V(\eta)= & \max _{\alpha}\{\alpha \eta d t-C(\alpha \eta) i d t+(1-i d t) \times \\
& \left.E_{d t}\left[V+V^{\prime} \cdot\left((i-\alpha) \eta_{t} d t+C\left(\alpha \cdot \eta_{t}\right) i d t+\zeta^{1 / 2} \eta^{1 / 2} d z_{t}\right)+\frac{1}{2} \zeta \eta V^{\prime \prime} d t\right]\right\}
\end{aligned}
$$

so that

$$
0=\max _{\alpha}\left\{\alpha \eta-C(\alpha \eta) i-i V+V^{\prime}((i-\alpha) \eta+C(\alpha \eta) i)+\frac{1}{2} \zeta \eta V^{\prime \prime}\right\} .
$$

This might be maximised at a corner value for $\alpha$, like $\alpha=0$, and one might further restrict $\alpha$ for small $\eta$ on grounds of solvency (to $0 \leq \alpha \leq i$ ) so that a second corner is introduced into consideration. If the optimal $\alpha$ arises as an internal point in the permitted range, then

$$
\eta-\eta i C^{\prime}(\alpha \eta)-\eta V^{\prime}+i \eta C^{\prime} V^{\prime}=0 .
$$

So provided $V^{\prime}(\eta) \neq 1$, the first order condition reads:

$$
C^{\prime}=1 / i
$$

Thus if $C(\cdot)$ is strictly convex, and the first order condition has a solution at all, the implied optimal dividend rate $\alpha \eta$ is constant, say $\bar{q}$. This is a remarkable conclusion and so calls for a brief interpretative inspection.

\section{Comparative statics for Bhattacharya's quadratic costs.}

If $C(x)=\gamma x^{2} / 2+\tau x$ is Bhattacharya's quadratic cost function, then the optimal dividend rate $\alpha \eta$ is given by

$$
\alpha \eta=\bar{q}=\left(\frac{1}{i}-\tau\right) / \gamma .
$$

This formula implies that (i) a lower tax rate on the dividend leads to a larger dividend, and this agrees with the usual tax-shelter intuition about retained earnings; (ii) higher costs associated with the cost of replacement funding (i.e. 
higher $\gamma$ ) lead to lower dividend payout; (iii) the higher the required rate of return on investment the lower is the dividend payout.

The corresponding equation for $V$ is

$$
\frac{1}{2} \zeta \eta V^{\prime \prime}+(i \eta-[\bar{q}-C(\bar{q}) i]) V^{\prime}-i V=C(\bar{q}) i-\bar{q} .
$$

As usual this has $V=\eta$ as a particular solution and the complementary equation is again a general confluent hypergeometric equation

$$
\frac{1}{2} \zeta \eta W^{\prime \prime}+(i \eta-[\bar{q}-C(\bar{q}) i]) W^{\prime}-i W=0,
$$

which is of the form

$$
\frac{1}{2} \zeta \eta W^{\prime \prime}+(i \eta-\bar{c}) W^{\prime}-i W=0,
$$

where $\bar{c}=\bar{q}-C(\bar{q}) i>0$, consistently with the sign in (9). This sign is positive since $C$ is convex, $C(0)=0$, and the choice $q=\bar{q}$ maximizes $q-C(q) i .^{12}$

Provided the frictions are not too large (say (22) holds), there exist monotonic solutions with qualitative behaviour the same as in the frictionless case. Assume therefore that frictions are low.

To find an instance of the optimal value function one would need to make some assumptions as to the nature of the options available to the firm. In general there is an abundance of solutions available depending on what structural assumptions one were to make about the firm.

For a qualitative illustration follow ACTW by assuming that only contraction in activity is available at any level of $\eta$. Thus only value functions that are defined by restriction from a decreasing non-negative function asymptotic to zero are considered. One must also assume that the value function has a known value when $\eta=0$. This known value is termed the dormant value by ACTW (and without loss of generality may be taken at unity).

So aiming towards a contradiction assume first that zero dividends are optimal near $\eta=0$, and let $P_{0}(\eta)$ be the standardized fundamental solution ${ }^{13}$ to the non-dividend equation (corresponding to $\kappa=1$ ) :

$$
\frac{1}{2} \zeta \eta P^{\prime \prime}+i \eta P^{\prime}-i P=0
$$

i.e. the solution subject to $P(0)=1$ and to $P(\eta)$ being asymptotic to zero at infinity.

One may then match any multiple $A_{0}$ of $P_{0}(\eta)$ to the solution of (26) by requiring continuity of value and of derivative at $\eta=\bar{\eta}$. Thus if $P_{\bar{q}}(\eta)$ is the

\footnotetext{
${ }^{12}$ In the case of Bhattacharya's quadratic

$$
\bar{c}=\bar{q}-C(\bar{q}) i=i \gamma \bar{q}+i \frac{\gamma}{2} \bar{q}^{2} .
$$

${ }^{13}$ In this section we drop earlier notation and let $P_{0}, P_{\bar{q}}, P_{i}$ describes various put-like functions (without the subscript implying values for $\kappa$ ).
} 
standardized fundamental solution of $(26)$ subject to $P_{\bar{q}}(0)=1$ one must solve for $A_{\bar{q}}$ and $\bar{\eta}$ the equations

$$
A_{\bar{q}} P_{\bar{q}}(\bar{\eta})=A_{0} P_{0}(\bar{\eta}), A_{\bar{q}} P_{\bar{q}}^{\prime}(\bar{\eta})=A_{0} P_{0}^{\prime}(\bar{\eta}) .
$$

So if $\bar{\eta}$ exists it is given by equating the corresponding elasticities ${ }^{14} \varepsilon_{\bar{q}}$ and $\varepsilon_{0}$ of the relevant put values, namely

$$
\varepsilon_{\bar{q}}=-\frac{P_{\bar{q}}^{\prime}(\bar{\eta})}{P_{\bar{q}}(\bar{\eta})}=-\frac{P_{0}^{\prime}(\bar{\eta})}{P_{0}(\bar{\eta})}=\varepsilon_{0} .
$$

These are non-zero. Substituting from the differential equations yields

$$
\frac{\bar{\eta}}{(\bar{\eta}-\bar{c} / i)}\left(\frac{\zeta}{2 i} \frac{P_{\bar{q}}^{\prime \prime}}{P_{\bar{q}}}-\frac{1}{\bar{\eta}}\right)=\frac{\zeta}{2 i} \frac{P_{0}^{\prime \prime}}{P_{0}}-\frac{1}{\bar{\eta}}
$$

Assuming the optimal value function is smooth $A_{\bar{q}} P_{\bar{q}}^{\prime \prime}(\bar{\eta})=A_{0} P_{0}^{\prime \prime}(\bar{\eta})$, and cancelling by the common non-zero factor, gives the following contradiction ${ }^{15}$.

$$
\frac{\bar{\eta}}{(\bar{\eta}-\bar{c} / i)}=1
$$

So turn now to a solution near the origin with an upper boundary on $\alpha$ of $\alpha=i$. Such a limitation on $\alpha \eta$ is the largest natural choice given (6), if one seeks at the extreme to ensure that the expected loss in value of $\eta$ is to be zero, i.e. $E[d \eta]=0$. If there are no frictions the solution is given by a decreasing modified Bessel function (see Appendix 5.3). When Bhattacharya's quadratic cost is invoked, this case leads to the more awkward equation

$$
\frac{1}{2} \zeta \eta V^{\prime \prime}+i\left(\gamma i^{2} \eta^{2} / 2+\tau i \eta\right) V^{\prime}-i V=i\left(\gamma i^{2} \eta^{2} / 2+\tau i \eta\right)-i \eta
$$

since $C(i \eta) i=i\left(\gamma i^{2} \eta^{2} / 2+\tau i \eta\right)$. As usual, a particular solution is $\eta$. Hence the complementary equation for a put-like function $P_{i}$ is

$$
\frac{1}{2} \zeta \eta P_{i}^{\prime \prime}+i\left(\gamma i^{2} \eta^{2} / 2+\tau i \eta\right) P_{i}^{\prime}-i P_{i}=0
$$

for which all (finite) points are regular singular. It is shown below that the qualitative properties of this equation are the same as the confluent hypergeometric equation. In fact, for $\eta$ small enough, the friction parameter $\gamma$ has negligible effect, as the equation may be approximated by

$$
\frac{1}{2} \zeta \eta V^{\prime \prime}+\tau i^{2} \eta V^{\prime}-i V=0
$$

\footnotetext{
${ }^{14}$ For an insight into the equation, observe that in the geometric Brownian case with $P(\eta)=$ $\eta^{-\kappa}$ the elasticity $-P^{\prime} / P$ is $\kappa / \eta$.

${ }^{15}$ Assuming tha $\eta>\bar{c} / i$ (i.e. the liquidation of assets services the perpetual bond $\bar{c}$ ) it is the case that $\eta /(\eta-\bar{c} / i)>1$, so if at a point of intersection of the two put-like functions the curvatures are nearly identical it follows that $\varepsilon_{\bar{q}}>\varepsilon_{0}$.
} 
or, using t he notation in (19) of section 4.1

$$
\eta V^{\prime \prime}+2 \lambda \tau i \eta V^{\prime}-2 \mu V=0 .
$$

Here $\lambda$ is being discounted down by the factor $i \tau$. Judging by the intuition of section 7.3 with $\lambda$ replaced by $\lambda \tau i$, it is optimal to choose $\alpha=i$ over $\alpha=0$, so this explains the earlier failure of smooth pasting $P_{0}$ and $P_{\bar{q}}$.

Now one can fit a scaled fundamental declining solution $P_{i}(\eta)$ of $(27)$ using value matching and smooth pasting at an unknown point $\hat{\eta}$. Thus one is led to solve

$$
A_{i} P_{i}(\hat{\eta})=A_{\bar{q}} P_{\bar{q}}(\hat{\eta}), A_{i} P_{i}^{\prime}(\hat{\eta})=A_{\bar{q}} P_{\bar{q}}^{\prime}(\hat{\eta}) .
$$

Here again the value of $\hat{\eta}$ is given by equating elasticities :

$$
\varepsilon_{\bar{q}}=-\frac{P_{\bar{q}}^{\prime}(\hat{\eta})}{P_{\bar{q}}(\hat{\eta})}=-\frac{P_{i}^{\prime}(\hat{\eta})}{P_{i}(\hat{\eta})}=\varepsilon_{i}
$$

Substituting from the differential equation this leads to

$$
\frac{i \hat{\eta}}{(i \hat{\eta}-\bar{c})}\left(\frac{\zeta}{2 i} \frac{P_{\bar{q}}^{\prime \prime}}{P_{\bar{q}}}-\frac{1}{\hat{\eta}}\right)=\frac{\hat{\eta}}{C(i \hat{\eta})}\left(\frac{\zeta}{2 i} \frac{P_{i}^{\prime \prime}}{P_{i}}-\frac{1}{\hat{\eta}}\right) .
$$

The usual continuity assumptions justify cancellation of a non-zero common factor to give

$$
\frac{i \hat{\eta}}{(i \hat{\eta}-\bar{c})}=\frac{\hat{\eta}}{C(i \hat{\eta})}
$$

i.e.

$$
\bar{c}=i \hat{\eta}-C(i \hat{\eta}) i
$$

But, by strict convexity, the maximum of $q-C(q) i$ is unique, so that

$$
i \hat{\eta}=\bar{q} \text {. }
$$

Thus the optimal policy switches continuously from $\alpha(\eta)=\bar{q} / \eta$ to $\alpha=i$. Despite the switch in dividend policy the overall qualitative behaviour of the value function at $\eta=\hat{\eta}$ is unaltered. But it is the case that pasting of two functions occurs ${ }^{16}$.

Turning now to a consideration of equation (27), observe that it is of the format

$$
y W^{\prime \prime}+y(A y+B) W^{\prime}-\mu W=0
$$

with $y=i \eta$ and $A, \mu>0$. The transformation $W=w \exp \left(-\frac{A}{4} y^{2}-\frac{B}{2} y\right)$ leads to the normalized form

$$
w^{\prime \prime}=J(y) w,
$$

\footnotetext{
${ }^{16}$ For $\eta>\bar{c} / i$ (and note that $i \eta=\bar{q}$ implies $\eta>\bar{c} / i$, since $\bar{q}>\bar{c}$ ) on heuristic grounds supposing that the curvature is much the same for each of the two put-like curves, one expects the relation $\varepsilon_{q} \geq \varepsilon_{i}$ to hold by virtue of

$$
\bar{c} \geq i \eta-i C(i \eta)
$$
}


where

$$
J(y)=\frac{\mu}{y}+\frac{1}{4}(A y+B)^{2}+\frac{A}{2}>0,
$$

and so $w(y)$ is convex for $y>0$. The argument from section 4.2 applies here also. Thus there are two non-negative, independent solutions with asymptotic behaviour

$$
w=y^{1 / 2} \exp \left( \pm \frac{A}{4} y^{2}\right) .
$$

Here we are applying the asymptotic formula for $y$ large

$$
w=J^{1 / 4}(y) \exp \left( \pm \int^{y} J^{1 / 2}(z) d z\right),
$$

see O'Neill (1991) or Bender and Orszag (1999). The Frobenius approach gives an indicial equation $c(c-1)=0$ and shows one solution to be a series $w_{1}=y+\ldots$ and the other $w_{2}=w_{1} \log y+\sum_{n=0}^{\infty} b_{n} y^{n}$. See Appendix 4. In this respect the solutions are thus qualitatively similar to those of the confluent hypergeometric equation with zero-coupon.

\section{Conclusion}

By reference to various generalizations of the ACTW paradigm it is shown that their simplified paradigm is justifiable, as a route to a valuable insight into the equity value of a firm. The approach of the current article offers a perspective on the behaviour of equity value at small and at large values of the aggregating variable. It allows the removal of an unrealistic cusp-like feature (vertical tangency of the curve at the origin) by introducing frictions. These frictions are also capable of rescuing the ACTW model from the criticism that their particular brand of put-like option is a potential source of instability, were the model to lead to parameter estimation studies. The current approach simplifies the analysis of comparative statics obtained by ACTW from numerical evidence by offering qualitative arguments. The discussion above argues the case for the inclusion of alternative options, including contraction as well as expansion options, calling for a classification of optimally managed firm types according to their embedded options, and by implication also calls for the construction of switching models that would exhibit all the qualitative patterns predicted by the smooth pasting of various confluent hypergeometric functions which solve the general valuation equation (including the snake example of Section 6). A preliminary analysis is offered of an endogenized selection of optimal dividend policy. No attempt was made here to search for new insights on such traditional questions as: solvency (bankruptcy constraints in the face of debt service), debt versus equity issuance (along the lines of Leland (1994) where the firm's embedded options would be expected to play some part in altering the optimal debt capacity), or of strategic aspects of dividend issue (compare the example of the Sealed Air Corporation's one-off exhaustive dividend issue as a committment 
tool - an interesting test case to try to accomodate in a theoretical development of dividend policy, see Wruck (1994)). 


\section{References}

Abramowitz, M.A, Stegan I.A, Handbook of mathematical functions, Dover 1972

Abel, A.B., Dixit, A.K., Eberley, J.C. and Pindyck, R.S., "Options, the Value of Capital, and Investment", Quarterly Journal of Economics,111(1996) 755-777

Ashton, D., Cooke, T.,Tippett, An Aggregation Theorem for the Valuation of Equity under Linear Information Dynamics, J. Business Finance \& Accounting,30 Nos 3\&4 (2003) 413-440

Ashton, D., Cooke,T., Tippett,M., Wang,P., Linear information Dynamics, Aggregation,Dividends and "Dirty Surplus" Accounting, to appear Accounting \& Business Research, 2004

Bender C.M, Orszag S.A., Advanced Mathematical Methods for Scientists and Engineers, Springer 1999

Bhattacharya S., Imperfect information, dividend policy, and "the bird in the hand" fallacy, Bell J. Economics, Vol 10 (1979) 259-270

Black F.; Cox, J.C., Valuing Corporate Securities: Some Effects of Bond Indenture Problems, JF Vol 31, No2, (May 1975) 351-367

Burgstahler, D., Dichev, I., Earnings, Adaptation and Equity Value, Accounting Review, 72,2,(1997) 187-215

Burgstahler, D., Discussion of "Combining Earnings and Book Value in Equity Valuation", Contemporary Accounting Research Vol 15 No. 3 (1998) 325341

Gietzmann,M.B.,Ostaszewski,A.J., Predicting Firm Value: The superiority of q-theory over residual income, Accounting \& Business Research 2004

Ince, E.L., Ordinary Differential Equations, Dover1956

Jeanblanc-Picque, M., Shiryaev,A.N., Optimization of the flow of dividends, Russ. Math. Surveys, 50 (1995) 257-277

Leland, H,E., Corporate Debt value, Bond covenants,, and Optimal Capital Structure, J. of Finance, Vol 49, No 4 (Sep. 1994)1213-1252

Merton, R.C., Theory of Rational Option Pricing, Bell J. of Economics and Management Science, 4 (1973) 141-183

Merton, R.C.,On the pricing of Corporate Debt: The Risk structure of Interest Rates, JF Vol 29, No 2, (May 1974) 449-470

Miller, J.C.P., On the choice of standard solutions for a homogeneous linear differential equation of the second order, Q.J. Mech. and Applied Math., Vol 3.(1950) 225-235

Miller, M.H., Modigliani, F., Dividend Policy, Growth, and the valuation of shares, J. of Business, Vol 34 (1961) 411-433

Ohlson, J.A., "Earnings, Book Values, and Dividends in Equity Valuation", Contemporary Accounting Research, 11, (1995) 661-687

Oksendal,B., Stochastic Differential Equations, Springer 1998

O’Neil P.V., Advanced Engineering Mathematics, Wadsworth 1991

Radner, R.,Shepp, L., Risk vs profit potential: A model for corporate strategy, J. Economic Dynamics \& Control, 20 (1996) 1371-1393 
Rochet,J.-C., Villeneuve, S., Liquidity risk and Corporate Demand for Hedging and Insurance, preprint, 2004

Russell, D.,"Mathematics of Finite Dimensional Control Systems", Dekker 1979

Slater, L.J., Confluent Hypergeometric Functions, Cambridge University Press, 1960

Wruck, K . H., Financial policy, internal control, and performance Sealed Air Corporation's leveraged special dividend, J. Fin. Eco. 36 (1994) 157 -192

Whittaker, E.T., Watson, G.N., Modern Analysis, Cambridge, 1946 


\section{Appendix 1: Proof of Proposition 1}

We use time labels 0 and 1 (the former for time $t=0$, the latter for time $t=\Delta t$ ); recall from (5) that assuming $\lim _{t \rightarrow \infty} e^{-i t} b_{t}=0$ we have

$$
\eta_{0}=b_{0}+\mathbf{E}\left[\int_{0}^{\infty} e^{-i t}(a+\varepsilon) d t\right] .
$$

Hence

$$
\begin{aligned}
& \eta_{0}=b_{0}+A_{0}+E_{0}=b_{0}+\mathbf{E}\left[\int_{0}^{\infty} e^{-i t} a d t+\int_{0}^{\infty} e^{-i t} \varepsilon d t\right] \\
& \eta_{1}=b_{1}+A_{1}+E_{1}=b_{1}+\mathbf{E}_{\Delta t}\left[\int_{\Delta t}^{\infty} e^{-i t} d a(t)+\int_{\Delta t}^{\infty} e^{-i t} d \varepsilon(t)\right]
\end{aligned}
$$

where $A=A(a)$ and $E=E(\varepsilon)$ denote the expected present values of future abnormal earnings and future adjustments given the information $a$ etc, so that $A_{0}=A\left(a_{0}\right)$ and so on.

To first order we thus have

$$
\begin{aligned}
& \eta_{0}-b_{0} \\
= & \mathbf{E}\left[\Delta A+\Delta E+(1-i \Delta t) \int_{0}^{\infty} e^{-i s} a^{\prime}(s) d s+(1-i \Delta t) \int_{0}^{\infty} e^{-i s} \varepsilon^{\prime}(s) d(s)\right] \\
= & \mathbf{E}\left[\Delta A+\Delta E+(1-i \Delta t)\left(A_{1}+E_{1}\right)\right], \\
= & a_{0} \Delta t+\varepsilon_{0} \Delta t+\mathbf{E}\left[(1-i \Delta t)\left(A_{1}+E_{1}\right)\right],
\end{aligned}
$$

Here $t=s-\Delta t$ and so $e^{-i t}=e^{-i s}(1-\Delta t)$ to first order; $a^{\prime}(s)=a(s+\Delta t)$ etc describes the shifted abnormal earnings process starting at $a_{1}=a(\Delta t)$, so that $A_{1}=A\left(a_{1}\right)$ etc. Subtracting the first two equations gives (to first order) and assuming $a$ and $\varepsilon$ are continuous processes

$$
\begin{aligned}
\mathbf{E}[\Delta \eta] & =\mathbf{E}\left[\eta_{1}\right]-\eta_{0} \\
& =\mathbf{E}\left[\Delta b-a_{0} \Delta t-\varepsilon_{0} \Delta t+i \Delta t\left(A_{0}+E_{0}-\Delta A-\Delta E\right)\right] \\
& =\mathbf{E}\left[\left(i b_{0}+a_{0}+\varepsilon_{0}\right) \Delta t-\Delta D-a_{0} \Delta t-\varepsilon_{0} \Delta t+i \Delta t\left(A_{0}+E_{0}\right)\right] \\
& =\mathbf{E}\left[i\left(b_{0}+A_{0}+E_{o}\right) \Delta t-\Delta D\right] \\
& =i \eta_{0} \Delta t-\Delta D .
\end{aligned}
$$

Appendix 2: Confluent Hypergeometric functions is

Recall the standard form of the confluent hypergeometric equation for $u(z)$

$$
u^{\prime \prime}+(B-z) u+A u=0 .
$$

The generalized Merton valuation equation is

$$
y w^{\prime \prime}+(y-c) w-\kappa w=0,
$$


from which setting $x=-y$ one obtains

$$
u^{\prime \prime}+(B-x) u+A u=0,
$$

with $A=-\kappa, B=-c$. The difficulty with applying standard solutions is that $u(-x)$ is liable to be undefined for $x>0$ on account of a branch cut (in the complex domain) for the logarithm function. It is therefore necessary to recompute the appropriate series solutions appropriate to the equation

$$
y \frac{d^{2} w}{d y^{2}}+(y+B) \frac{d w}{d y}+A y=0,
$$

for $y>0$. A summary of results follows. The notation just adopted here is to help the reader compare formulas here against those in Slater (1960).

\section{A2.1 Summary of some useful results}

The notes show in summary that we have the following solution formats ( $m$ is a positive integer).

0. $A=B=-m$

$$
K\left(1-y+\frac{y^{2}}{2 !}-\ldots+\frac{(-1)^{m}}{m !} y^{m}\right)+L e^{-y} .
$$

Note that the first function is obtainable by the method of reduction of order; see the footnote ahead of equation (23).

1. Provided $B \notin\{0,-1,-2, \ldots\}$

$$
M(A, B,-y)=1-\frac{A}{B} y+\frac{1}{2} \frac{A(A+1)}{B(B+1)} y^{2}+\ldots
$$

2. Provided $B \notin\{1,2, \ldots\}$ a solution is given by

$$
(-y)^{1-B} M(A-B+1,2-B,-y) .
$$

For $B=0$ this includes polynomial solutions with a root at the origin when $A$ is a negative integer. by

3. For $B=-m$ and $A \notin\{0,-1, \ldots,-m\}$ there is a regularized solution given

$$
\bar{M}(A, B,-y)=(m+1) ! a_{m+1}\left(\frac{y^{m+1}}{(m+1) !}-\frac{(A+m+1)}{(m+2) !(1)} y^{m+2}+\ldots\right) .
$$

4. For $B=-m<0$ and $A=-k$ with $k<m$ and $A \neq B$ (thus $A>B$ ) we have the polynomial solution

$$
a_{0}\left(1-\frac{A}{B} y+\ldots+\frac{(A) \ldots(-2)(-1)}{k !(B) \ldots(B+k-1)} y^{k}\right) .
$$


5. For $B=-m$ and $A \notin\{0,-1, . .,-m\}$

$$
\begin{aligned}
& \bar{M}(A, B,-y) \log |y|+\left(1-\frac{A}{B} y+\ldots+\frac{A(A+1) \ldots(A+m-1)}{m ! B(B+1) \ldots(B+m-1)} y^{m}\right) \\
& +b_{m+1}\left(y^{m+1}-\frac{(A+m+1)}{m+2} y^{m+2} \ldots\right)+\frac{a_{1-B}}{(2-B)}\left[u_{m+2} y^{m+2}+\ldots\right],
\end{aligned}
$$

where

$$
a_{1-B}=\frac{(A)_{1-B}}{(B)_{-B}(1-B) !}
$$

and $(A)_{m}=(A+1) \ldots(A+m-1)$ is the Pochammer symbol. When $m=0$ and $A=-\kappa$ this is interpreted as saying that

$$
\bar{M}(-\kappa, 0,-y) \log |y|+1+b_{1}\left(y+\frac{(\kappa-1)}{2} y^{2}+\ldots\right)+\frac{a_{1}}{2}\left[u_{2} y^{2}+\ldots\right] .
$$

See also Slater (1960: 8) formula (1.5.18).

\section{Appendix 3: The case $\lambda=0$}

The case when $\alpha=i$ corresponds to the limiting case $\kappa=\infty$, or more properly to the parameter value $\lambda=0$. This case is also of significant interest - see section 8 above. To characterize the non-negative equity value functions, we look to the differential equation (20), which now reads

$$
\eta \frac{d^{2} W}{d \eta^{2}}=2 \mu W
$$

This may be recast more simply: putting $y=2 \mu \eta$ with say $R(y)=R(2 \mu \eta)=$ $W(\eta)$ one obtains

$$
y R^{\prime \prime}=R,
$$

a Schrödinger equation of order 2. It is well-known (see Bender and Orszag (1999: 88) that the asymptotics for large $y$ are given as one might expect by

$$
R(y) \sim C y^{-1 / 4} \exp \left( \pm y^{1 / 2}\right) .
$$

The series solution for the increaing solution is

$$
R(y)=y+\frac{y^{2}}{2}+\frac{y^{3}}{12}+\ldots+\frac{y^{n}}{n !(n-1) !}+\ldots .
$$

It is of course given by the modified Bessel functions of order one:

$$
\frac{\sqrt{x}}{2} I_{ \pm 1}\left(\frac{\sqrt{x}}{2}\right)
$$

or

$$
\frac{\sqrt{x}}{2} K_{1}\left(\frac{\sqrt{x}}{2}\right)
$$




\section{Appendix 4: Solution of a related differential equation}

We consider the following equation which arises in Section 8 .

$$
\eta V^{\prime \prime}+\frac{i}{\zeta} i \eta(\gamma i \eta+2 \tau) V^{\prime}-\frac{2 i}{\zeta} V=0
$$

Putting $y=i \eta$

$$
i \eta \frac{V^{\prime \prime}}{i^{2}}+\frac{i}{\zeta} i \eta(\gamma i \eta+2 \tau) \frac{V^{\prime}}{i}-\frac{2}{\zeta} V=0
$$

write this in the form

$$
y W^{\prime \prime}+\frac{i}{\zeta} y(\gamma y+2 \tau) W^{\prime}-\frac{2}{\zeta} W=0 .
$$

We therefore study the form

$$
y W^{\prime \prime}+y(A y+B) W^{\prime}-\mu W=0 .
$$

We put

$$
W=\sum_{n=0}^{\infty} a_{n} x^{n+c}
$$

yielding

$$
\begin{aligned}
0= & \sum_{n=0}^{\infty}(n+c)(n+c-1) a_{n} x^{n+c-1}+A \sum_{n=0}^{\infty}(n+c) a_{n} x^{n+1+c} \\
& +B \sum_{n=0}^{\infty}(n+c) a_{n} x^{n+c}-\mu \sum_{n=0}^{\infty} a_{n} x^{n+c} .
\end{aligned}
$$

The indicial equation (at $x^{c-1}$ ) is

$$
c(c-1)=0
$$

The next power is $x^{c}$ and yields the equation

$$
c(c+1) a_{1}=(\mu-B c) a_{0} .
$$

Opting for the larger root $c=1$ gives

$$
\sum_{n=0}^{\infty}(n+1) n a_{n} x^{n}+A \sum_{n=0}^{\infty}(n+1) a_{n} x^{n+2}+B \sum_{n=0}^{\infty}(n+1) a_{n} x^{n+1}-\mu \sum_{n=0}^{\infty} a_{n} x^{n+1}=0 .
$$

Consideration of the coefficient at $x^{n+1}$ yields

$$
a_{n+1}(n+2)(n+1)-A n a_{n-1}+B(n+1) a_{n}-\mu a_{n}=0,
$$


SO

$$
a_{n+1}(n+2)(n+1)=(\mu-B(n+1)) a_{n}+A n a_{n-1},
$$

with

$$
a_{1}=\frac{\mu-B}{2} a_{0} .
$$

Hence the series has infinite radius of convergence. Note that

$$
6 a_{2}=A a_{0}-(2 B-\mu) a_{1}=A a_{0}+(\mu-B)(\mu-2 B) a_{0} .
$$

The Frobenius theory implies that in this case the second solution is logarithmic and of form

$$
k(x+\ldots) \log x+\sum_{n=0}^{\infty} b_{n} x^{n}
$$

exhibiting a cusp-like feature at the origin. See O'Neil (1991: 326).

\section{Appendix 5: The Incomplete Laplace Transform approach}

Since $V^{\prime}(0+)$ is liable to be infinite for some solutions of Kummer's equation, one should employ the incomplete transform:

$$
L_{\delta}\{f\}=\int_{\delta}^{\infty} f(t) e^{-s t} d t
$$

with the associated three rules

$$
\begin{aligned}
L_{\delta}\{t f(t)\} & =-\frac{d}{d s} L_{\delta}\{f\}, \quad L_{\delta}\left\{f^{\prime}\right\}=s L_{\delta}\{f\}-f(\delta) e^{-s \delta}, \\
L_{\delta}\left\{f^{\prime \prime}\right\} & =s^{2} L_{\delta}\{f\}-f(\delta) s e^{-s \delta}-f^{\prime}(\delta) e^{-s \delta} .
\end{aligned}
$$

Writing $\tilde{f}=L_{\delta}\{f\}$ these rules lead on application to the standardized ACTW equation (21) to

$$
\tilde{f}^{\prime}+\frac{2 s+\kappa+1}{s(s+1)} \tilde{f}=\frac{1}{s(s+1)}\left[f(\delta) e^{-s \delta}+\delta f(\delta)(1-s) e^{-s \delta}-\delta f^{\prime}(\delta) e^{-s \delta}\right] .
$$

The integrating factor is $s^{\kappa+1}(1+s)^{1-\kappa}$ and integration from unity to $s$ yields

$$
s^{\kappa+1}(1+s)^{1-\kappa}(\tilde{f}(s)-\tilde{f}(1))=\left[f(\delta)-\delta f^{\prime}(\delta)\right] I_{1}(\delta)+\delta f(\delta) I_{2}(\delta),
$$

where

$$
I_{1}(\delta)=\int_{1}^{s} u^{\kappa}(1+u)^{-\kappa} e^{-u \delta} d u, \quad I_{2}(\delta)=\int_{1}^{s} u^{\kappa}(1+u)^{-\kappa}(1-u) e^{-u \delta} d u
$$

Series solution considerations yield $\lim _{\delta \rightarrow 0} \delta f(\delta)=\lim _{\delta \rightarrow 0} \delta f^{\prime}(\delta)=0$. As $e^{-u \delta}$ is decreasing in $\delta$ it is the case that $I_{j}(\delta) \leq I_{j}(0)$, and so passing to the limit as 
$\delta \rightarrow 0$, the solution to the differential equation is given by the function whose transform is

$$
\frac{\tilde{f}(s)}{f(0)}=\frac{(1+s)^{\kappa-1}}{s^{\kappa+1}} \int_{1}^{s} u^{\kappa}(1+u)^{-\kappa} d u+C \frac{(1+s)^{\kappa-1}}{s^{\kappa+1}},
$$

where $C$ is a constant. A computation for the two cases $\kappa=1,2$ follows.

A 5.1 Laplace transform approach when $\kappa=1$

Here the solution satisfies

$$
\tilde{f}(s)=s^{-2} \int_{1}^{s} \frac{u d u}{u+1}+\frac{C}{s^{2}}=-\frac{1}{s^{2}} \log (1+s)+\frac{1}{s}+\frac{C^{\prime}}{s^{2}} .
$$

Now refer to Euler's exponential integral and its asymptotic expansion

$$
E_{1}(x)=\int_{1}^{x} \frac{e^{-u x} d u}{u}=\frac{e^{-x}}{x}\left\{1-\frac{1}{x}+\frac{2}{x^{2}}+\ldots\right\} .
$$

This has transform

$$
\frac{1}{s} \log (1+s),
$$

so that

$$
L\left\{t E_{1}(t)\right\}=-\frac{d}{d s}\left\{\frac{1}{s} \log (1+s)\right\}=-\frac{1}{s^{2}} \log (1+s)-\frac{1}{s(s+1)} .
$$

Using partial fractions, the conclusion is that

$$
\frac{\tilde{f}(s)}{f(0)}=\left[-\frac{1}{s^{2}} \log (1+s)-\frac{1}{s(s+1)}\right]+\frac{2}{s}-\frac{1}{s+1}+\frac{C^{\prime}}{s^{2}} .
$$

The solution to the ode thus takes the form

$$
t E_{1}(t)+2-e^{-t}+K t .
$$

Note that

$$
E_{1}(t)=-\gamma-\log t+\sum \frac{(-1)^{n-1} t^{n}}{n ! n},
$$

so this result is in agreement with the series expansion.

A 5.2 Laplace transform approach when $\kappa=2$

Here the solution obeys

$$
\begin{aligned}
\frac{\tilde{f}(s)}{f(0)} & =\frac{1+s}{s^{3}} \int_{1}^{s}\left(\frac{u}{1+u}\right)^{2} d u+C \frac{(1+s)}{s^{3}} \\
& =\frac{1+s}{s^{2}}-\frac{1}{s^{3}}-\frac{2(1+s) \log (1+s)}{s^{3}}+C\left(\frac{1}{s^{3}}+\frac{1}{s^{2}}\right),
\end{aligned}
$$


or, since $u /(1+u)=1-1 / v$ with $v=1+u$, and ignoring constants absorbed by $C$,

$$
\begin{aligned}
\int\left(\frac{u}{1+u}\right)^{2} d u & =\int\left(1-\frac{2}{1+u}+\frac{1}{(1+u)^{2}}\right) d u \\
& =s-2 \log (1+s)-\frac{1}{1+s}
\end{aligned}
$$

But

$$
L\left\{\left(t^{2}+2 t\right) E_{1}(t)\right\}=-\frac{2(1+s) \log (1+s)}{s^{3}}-\left(\frac{3}{s}-\frac{3}{s+1}\right)+\frac{1}{(1+s)^{2}} .
$$

Thus the solution is a multiple of

$$
f(t)=C\left(\frac{t^{2}}{2}+t\right)+\left(t^{2}+2 t\right) E_{1}(t)+1+t-\frac{t^{2}}{2}+3\left(1-e^{-t}\right)-t e^{-t}
$$

A 5.1 Laplace transform approach when $\kappa=\infty$

Here the equation to solve is

$$
\tilde{f}^{\prime}+\frac{2 s+1}{s^{2}} \tilde{f}=\frac{f(0)}{s^{2}} .
$$

The integrating factor is $s^{2} e^{-1 / s}$ so

$$
\frac{\tilde{f}(s)}{f(0)}=\frac{e^{1 / s}}{s^{2}} \int^{s} e^{-1 / u} d u+C \frac{e^{1 / s}}{s^{2}} .
$$

Since

$$
\frac{e^{1 / s}}{s^{2}}=\frac{1}{s^{2}}+\frac{1}{s^{3}}+\frac{1}{2 s^{4}}+\ldots+\frac{1}{n ! s^{n+2}}+\ldots
$$

and on the one hand is a tabulated transform of

$$
\sqrt{t} I_{1}(\sqrt{t})
$$

involving the modified Bessel function $I_{\nu}(x)$, but is on the other hand the transform of the series

$$
t+\frac{t^{2}}{2}+\frac{t^{3}}{12}+\ldots
$$

Note that the modified Bessel equation of order $\nu$ takes the form (Abramowitz and Stegun (1970): 374)

$$
x^{2} w^{\prime \prime}+x w^{\prime}-\left(x^{2}+\nu^{2}\right) w=0,
$$

with solutions $I_{ \pm \nu}(x)$ and $K_{\nu}(x)$. When $\nu=1$ the substitution $y=\sqrt{x} w(\sqrt{x})$ yields

$$
x y^{\prime \prime}=\frac{1}{4} y .
$$


The further substitution $x=4 t$ and $z(t)=y(4 t)$ leads to $t z^{\prime \prime}(t)=z$, so the solution of the latter is seen to be

$$
z(t)=y(x / 4)=\frac{\sqrt{x}}{2} w\left(\frac{\sqrt{x}}{2}\right),
$$

a well-known result. (See Abramowitz and Stegun (1970) page 374 for graphs, and page 506 for the limiting case of the confluent functions.) 